\title{
Nanobiotechnology medical applications: Overcoming challenges through innovation
}

\author{
Anthony Singer ${ }^{1,2,4}$, Eleni Markoutsa ${ }^{1,2,4}$, Alya Limayem ${ }^{1,4}$, Subhra Mohapatra ${ }^{3,4,5}$ \\ and Shyam S. Mohapatra ${ }^{1,2,4,5 *}$
}

\begin{abstract}
Biomedical Nanotechnology (BNT) has rapidly become a revolutionary force that is driving innovation in the medical field. BNT is a subclass of nanotechnology (NT), and often operates in cohort with other subclasses, such as mechanical or electrical NT for the development of diagnostic assays, therapeutic implants, nano-scale imaging systems, and medical machinery. BNT is generating solutions to many conventional challenges through the development of enhanced therapeutic delivery systems, diagnostic techniques, and theranostic therapies. Therapeutically, BNT has generated many novel nanocarriers (NCs) that each express specifically designed physiochemical properties that optimize their desired pharmacokinetic profile. NCs are also being integrated into nanoscale platforms that further enhance their delivery by controlling and prolonging their release profile. Nano-platforms are also proving to be highly efficient in tissue regeneration when combined with the appropriate growth factors. Regarding diagnostics, NCs are being designed to perform targeted delivery of luminescent tags and contrast agents that enhance the NC -aided imaging capabilities and resulting diagnostic accuracy of the presence of diseased cells. This technology has also been advancing the ability for surgeons to practice true precision surgical techniques. Incorporating therapeutic and diagnostic NC-components within a single NC can facilitate both functions, referred to as theranostics, which facilitates real-time in vivo tracking and observation of drug release events via enhanced imaging. Additionally, stimuli-responsive theranostic NCs are quickly developing as vectors for tumor ablation therapies by providing a model that facilitates the location of cancer cells for the application of an external stimulus. Overall, BNT is an interdisciplinary approach towards health care, and has the potential to significantly improve the quality of life for humanity by significantly decreasing the treatment burden for patients, and by providing non-invasive therapeutics that confer enhanced therapeutic efficiency and safety.
\end{abstract}

Keywords: Biomedical nanotechnology, nanotherapeutics, nanodiagnostics, theranostics, nanocarriers

${ }^{1}$ College of Pharmacy Graduate Programs, University of South Florida, Tampa, FL, USA

${ }^{2}$ Department of Internal Medicine

${ }^{3}$ Department of Molecular Medicine

${ }^{4}$ Center for Research and Education in Nanobioengineering, Morsani College of Medicine University of South Florida, Tampa, FL, USA

${ }^{5}$ James A. Haley VA Hospital, Tampa, FL, USA

*Corresponding author: S. S. Mohapatra E-mail: smohapat@health.usf.edu

DOI: 10.2478/ebtj-2018-0019

(C) 2018 Authors. This work was licensed under the Creative Commons AttributionNonCommercial-NoDerivs 3.0 License.

\section{Introduction}

Nanotechnology (NT) is a dynamic revolutionary force that is exponentially impacting societies and environments globally. Through ground-breaking scientific and technological advancements, NT-based products have become integrated into the daily lives of nearly every human on the planet. The NT revolution truly embodies the interdisciplinary approach and is bringing scientists together from nearly every area of the scientific study. This world-wide collaboration is significantly enhancing the quality of construction materials, machinery, automobiles, electronics, renewable energy, transportation, common appliances, consumer products, entertainment, agriculture, microscopy, scientific instrumentation, health care, diagnostic assays, and medicinal drug discovery (1-5) (Fig. 1). NTs exemplify unique physiochemical properties that present novel functionality based upon their extremely small size, large surface area, and quantum-mechanical dictation $(1,3-5)$. Although the benefits are clear, there are growing concerns about the short and long-term impact of the rapid NT expansion on the health and safety of humans and the protection of the environment $(1,3,4)$. To address these concerns, the NT community is working to generate regulations for nanofabrication, nano-synthesis and waste re- 


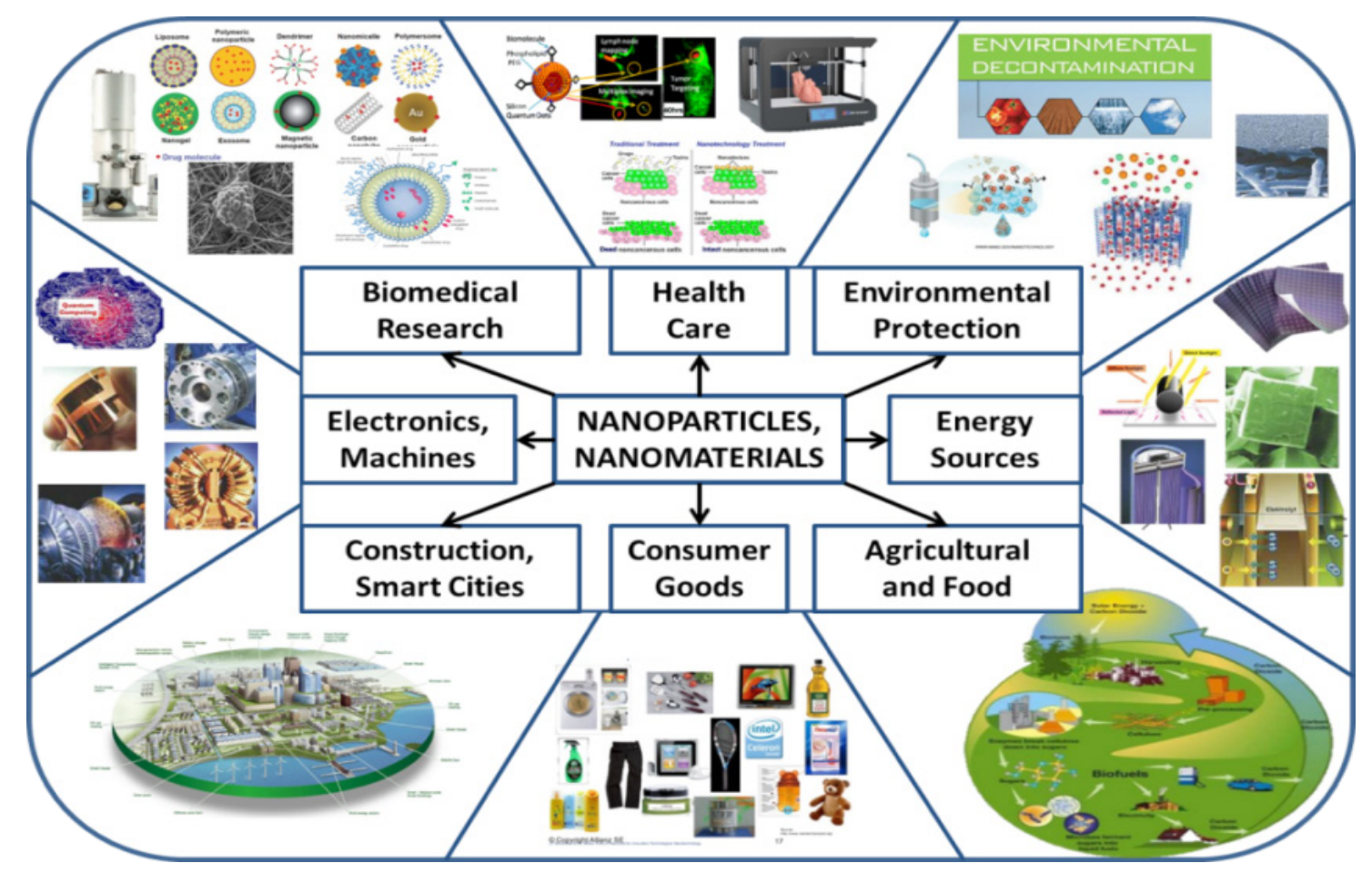

Figure 1. Nanoparticle and Nanomaterial Applications: Biomedical Research; Health Care; Environmental Protection; Energy Sources; Agriculture and Food; Consumer Goods; Construction, Smart Cite; and Electronics, Machines. (top left corner, then clockwise).

moval for the available nanoparticles (NPs) and nanomaterials (NMs) $(2,3)$. These additions to the NT revolution will aid in the development of products with the following properties: non-toxic, biodegradable, biocompatible, scalable, smart or programmable, eco-friendly, low energy consuming, and highly efficient $(4,5)$. Therefore, the future of NT has the potential to generate outstanding medical care, extremely powerful computational power, stronger and more durable machinery, eco-friendly cities, and an increase in the access to clean water and food $(3,4)(6-8)$.

One of the most prominent subclasses of NT is Biomedical NT (BNT), which focuses on the development of nano-based therapeutics, therapies, and diagnostic techniques that will advance health care. Currently, only a few nanomedicines out of the thousands in research and development $(\mathrm{R} \& \mathrm{D})$ are FDA approved, but many BNT-based applications are being applied to the laboratory setting, such as diagnostic assays, imaging technologies, biosensors, and in vitro tissue generation (9). These innovations are enhancing the accuracy and precision of scientific research by lowering the detection limits, enabling high-throughput screening (HTS) capabilities, developing bio-mimetic in vitro and in vivo models, and allowing researchers to visualize and track their nano-products within living organisms and environmental landscapes. Through the application of these models, lead selection and optimization during the R\&D of nanomedicine will be greatly enhanced, which will increase the therapeutic efficiency of the final product. Overall, the current applications of BNT are significantly enhancing scientific research, which is giving rise to precision and personalized nano-based therapies and health care.

Personalized therapies are appearing to be the next major trend in the BNT field, and will primarily focus on selecting the dose type, level and strength based upon a patient's specific disease profile. This advancement will heavily depend on the simultaneous generation of comprehensive genomic, proteomic, metabolomics, and cytomic databases that can be used to analyze results and select the proper protocols for therapies. These omic-based studies will facilitate accurate disease diagnosis and therapy selection that will prove to be vital to the advancement of curative personalized medicine therapies for extremely high-mortality rate diseases, such as cardiovascular and neurodegenerative diseases. Another example can be seen in the development of personalized cancer therapies that will be dictated by the cross-referencing of acquired patient data with cancer-omic databases that contain information regarding the molecular or genetic carcinogenic agents, cell morphology, surface and genomic biomarkers, protein expression, symptoms, and alterations to metabolic pathways (10-12). By tailoring the design of nanoformulations to the omic-based information, they can be created to express prolonged half-life, controlled drug release, targeted delivery, stimuli-responsive behavior, and enhanced permeability across physiological barriers. Nanomedicine has quickly surpassed the capabilities of conventional pharmaceuticals. In the past, the majority of therapies were able to efficiently manage diseases, but typically did not offer the curative options that are now becoming a possibility through the BNT advancement.

\section{Nanotherapies: State of the art}

Nanomedicines are designed to avoid inducing complications that a patient would typically experience with conventional pharmaceuticals, for example, toxicity induced from the administration of large, non-targeted dosages. However, nano- 


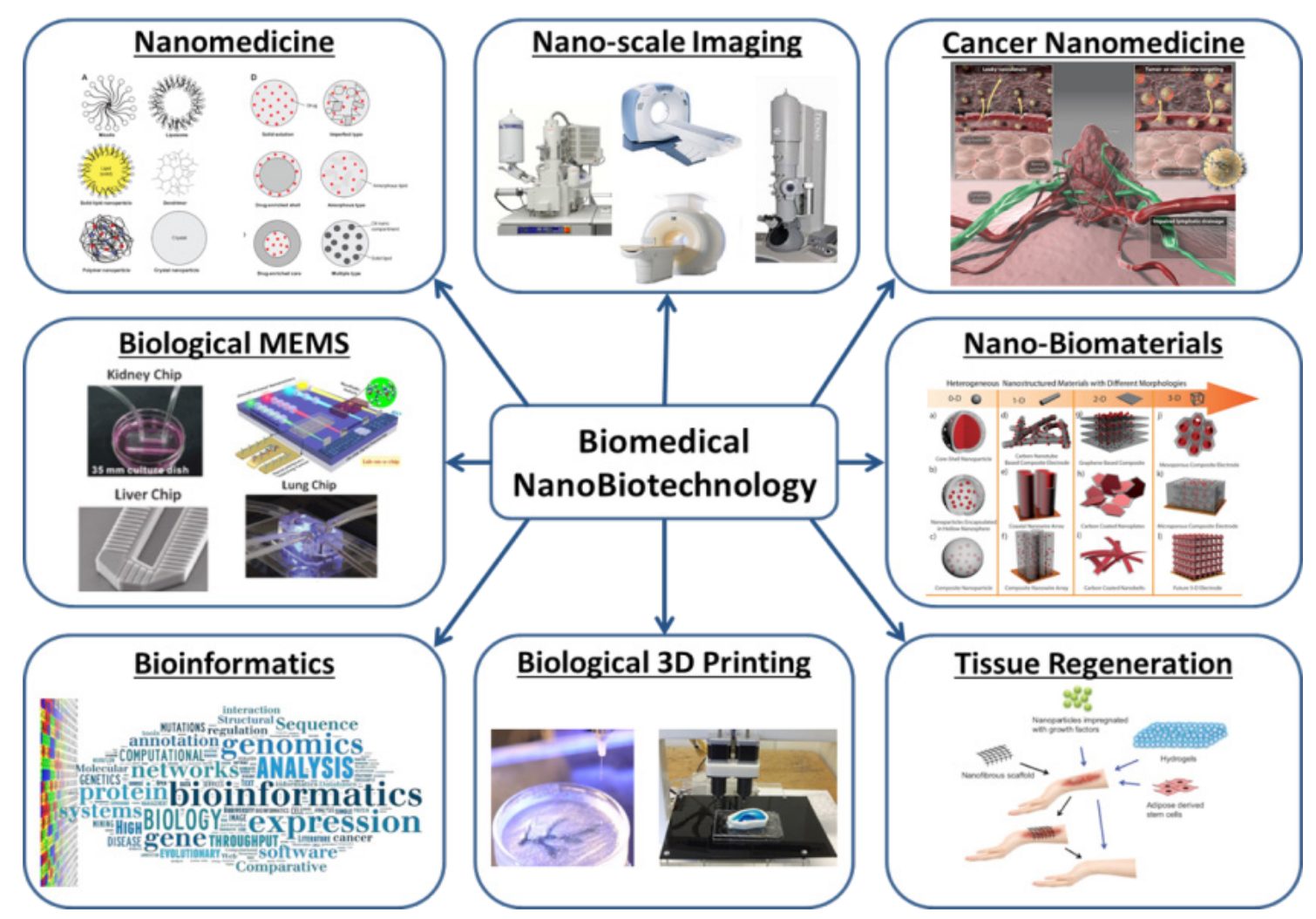

Figure 2. Biomedical Nanotechnology Applications: Nano-scale Imaging; Cancer Nanomedicine; Nano-Biomaterials; Tissue Regeneration; Biological 3D Printing; Bioinformatics; and Biological MEMS (top left corner, then clockwise).

medicines can typically be administered at much smaller doses due to their innately high reactivity and functionalized drug delivery. Nanomedicines are often loaded within or adhered to functionalized NCs that confer enhanced therapeutic efficiency. The functionalization of NCs incorporates bioactive molecules that facilitate control over the targeted delivery and release of the payload, the selective and efficient permeation across targeted physiological barriers, and the safe excretion and degradation within living organisms (13-16). These nanomedicines are beginning to be generated to treat a wide variety of health issues, including high mortality rate diseases like cancer, cardiovascular disease, and neurodegenerative disease (Table 1). Some examples of highly effective nano-therapies currently in $\mathrm{R} \& \mathrm{D}$ or clinical trials include tumor ablation through hyperthermia, phototherapy, or photo-chemotherapy; cardiovascular therapies utilizing targeting nanomedicines directed to sites of thrombosis and atherosclerotic plaques; and neurodegenerative therapies utilizing nanomedicines that can permeate the blood-brain barrier (BBB) in order to inhibit the progression, prevent the genesis, or possibly revert the symptoms of Parkinson's and Alzheimer's disease. As these BNT-enabled innovations continue to develop, many classically chronic diseases will begin to become curable.

Recently, many NCs are being functionalized with extracellular or intracellular stimuli-responsive behaviors that are stimulated upon interaction with a selected biomolecule or cellular environment that causes a structural or conformational change in the NC, thus inducing payload release or bioactivity
$(17,18)$. Common stimuli found in the cellular microenvironment of most disease sites includes detectable alterations from endogenous $\mathrm{pH}$, temperature, and glutathione levels (19). Extracellular stimuli can also be applied, which include induced alternating magnetic fields, photothermal energy (NIR laser), and photoacoustic waves (20). Induced changes in the NC can range from alterations in porosity to the cleavage of molecular linkers to activate a specific functionality. Techniques like these have been shown to significantly enhance the therapeutic efficiency of in vivo administration by avoiding potentially toxic non-specific interactions or premature drug release, while also ensuring that an adequate concentration of drug is delivered to the target site $(18,20-23)$.

\section{Organic Nanocarriers}

Novel NCs are constantly in demand, and through vigorous BNT research, many have been discovered and are currently under investigation for their potential to enhance the delivery of conventional payloads or novel nanomedicines. A very common form of NCs are those made of natural polymers, which includes proteins and lipids. Polymeric NCs are biocompatible, biodegradable, and non-toxic which makes them ideal for medical application. Additionally, they can be highly functionalized in a manner that facilitates novel functionalities, and control over the therapeutic delivery and release profiles. A novel example can be seen in a recently developed star-shaped poly (l-lysine) polypeptide (star-PLL) NC that has been shown to facilitate enhanced gene therapy via highly efficient intra- 
Table 1. Novel Nanocarrier-based Drug Delivery Systems (Section 2.0)

\begin{tabular}{|c|c|c|c|c|c|}
\hline & Type & Material & $\begin{array}{l}\text { Novel Physiochemical } \\
\text { Properties }\end{array}$ & Novel Therapeutic Applications & Ref \\
\hline \multicolumn{6}{|c|}{ Organic Nanocarriers (Section 2.1) } \\
\hline 1 & Polymeric & Star-PLL & $\begin{array}{l}\text { - Stable internalization } \\
\text { facilitates intracellular release } \\
\text { of pDNA }\end{array}$ & $\begin{array}{l}\text { - Stimulates tissue regeneration } \\
\text { - Utilizes lower doses of pDNA while maintaining } \\
\text { efficient expression levels }\end{array}$ & 24 \\
\hline 2 & Polymeric *** & $\begin{array}{l}\text { PEG-chain } \\
\text { Micelle }\end{array}$ & $\begin{array}{l}\text { - } \mathrm{pH} \text {-responsive release of } \\
\text { pro-drug at thrombosis }\end{array}$ & - Enhanced subsequent anti-thrombotic therapy & 25 \\
\hline 3 & Lipid Micelles & Chitosan coated & $\begin{array}{l}\text {-Cationic Charge } \\
\text {-Thiolation-enhanced } \\
\text { mucoadhesiveness }\end{array}$ & $\begin{array}{l}\text {-Enhanced delivery of nucleic acids } \\
\text {-Enhanced retention and permeability within } \\
\text { mucosal membranes }\end{array}$ & $26-29$ \\
\hline 4 & Sertoli Cells & $\begin{array}{l}\text { Naturally de- } \\
\text { rived cells }\end{array}$ & -Encapsulation of lipid micelles & $\begin{array}{l}\text {-Enhanced immunoprotection and delivery to the } \\
\text { lung }\end{array}$ & 30 \\
\hline 5 & Liposomes & $\begin{array}{l}\text { Phospholipid } \\
\text { bilayer }\end{array}$ & $\begin{array}{l}\text { - Formation of } \\
\text { immunoliposomes }\end{array}$ & $\begin{array}{l}\text { - Cancer immunity therapies } \\
\text { - Delivery of nanoprimers }\end{array}$ & 31 \\
\hline 6 & Nanobubbles $* * *$ & $\begin{array}{l}\text { Phospholipid } \\
\text { bilayer }\end{array}$ & $\begin{array}{l}\text { - Externally induced } \\
\text { ultrasonic-responsive behavior }\end{array}$ & $\begin{array}{l}\text { - Enhanced tumor-permeability through induced } \\
\text { structural cavitation }\end{array}$ & 34 \\
\hline 7 & Exosomes & cell-derived & $\begin{array}{l}\text { - Biocompatible surface chemis- } \\
\text { try to originator cells, enhanced } \\
\text { targeting }\end{array}$ & $\begin{array}{l}\text { - Laboratory stimulated biogenesis for their } \\
\text { utilization as biomimetic nanocarrier }\end{array}$ & $35-38$ \\
\hline \multicolumn{6}{|c|}{ Inorganic Nanocarriers (Section 2.2) } \\
\hline 8 & Mesoporous Silica & $\begin{array}{l}\text { Si-Ph, Si-N, } \\
\text { Si-OH }\end{array}$ & $\begin{array}{l}\text { - Tunable porosity controls } \\
\text { loading \& release }\end{array}$ & $\begin{array}{l}\text { - Enhanced delivery of anti-malarial, \& anti-leukemic } \\
\text { therapeutics }\end{array}$ & 39,40 \\
\hline 9 & $\begin{array}{l}\text { Gold Nanoparti- } \\
\text { cles (AuNPs) }\end{array}$ & $\mathrm{Au}$ & $\begin{array}{l}\text { - polyvinylpyrrolidone conju- } \\
\text { gates for enhanced internal- } \\
\text { ization }\end{array}$ & $\begin{array}{l}\text { - Non-toxic intracellular co-delivery of DOX \& BLM to } \\
\text { lung cancer cells }\end{array}$ & 41,42 \\
\hline 10 & $\begin{array}{l}\text { Metal Organic } \\
\text { Frameworks }\end{array}$ & $\begin{array}{l}\text { Alumi- } \\
\text { num-based }\end{array}$ & $\begin{array}{l}\text { - Functional and structural } \\
\text { tenability within lysosomal } \\
\text { low-pH environment }\end{array}$ & $\begin{array}{l}\text { - Deliver antioxidant for up to one week while } \\
\text { lysosomally encapsulated }\end{array}$ & 43 \\
\hline 11 & ZIFs $* * *$ & $\begin{array}{l}\text { Zeolitic Imidaz- } \\
\text { olate }\end{array}$ & $\begin{array}{l}\text { - High degree of biocompati- } \\
\text { bility enhanced drug biodistri- } \\
\text { bution }\end{array}$ & $\begin{array}{l}\text { - Release anti-cancer agents upon interaction with } \\
\text { acidic } \mathrm{pH} \text { or the tumor microenvironment }\end{array}$ & 44 \\
\hline \multicolumn{6}{|c|}{ Nano-platforms (Section 2.3) } \\
\hline 12 & $\begin{array}{l}\text { Ocular } \\
\text { Nanowafers }\end{array}$ & Polymeric & $\begin{array}{l}\text { - Structure has drug embedded } \\
\text { throughout nanowafer, and } \\
\text { slowly degrades via hydrolysis }\end{array}$ & $\begin{array}{l}\text { - Achieve prolonged gradual release of ocular drug, } \\
\text { improves ocular bioavailability \& therapeutic effi- } \\
\text { ciency }\end{array}$ & 45,46 \\
\hline 13 & $\begin{array}{l}\text { Dental Adhesive } \\
\text { Nano-resins }\end{array}$ & $\begin{array}{l}\text { Nano-scale } \\
\text { polymer }\end{array}$ & $\begin{array}{l}\text { - Biocompatible structure, and } \\
\text { enhanced adhesion }\end{array}$ & - Self-cleaning and self-healing restorative methods & 3 \\
\hline 14 & $\begin{array}{l}\text { Microneedle } \\
\text { Patches }\end{array}$ & Alginate-based & $\begin{array}{l}\text { - MNs increase transdermal } \\
\text { permeability }\end{array}$ & $\begin{array}{l}\text { - Glucose-responsive release of exendin- } 4 \text { for pro- } \\
\text { longed, on-demand Type-2 Diabetes therapy }\end{array}$ & 47 \\
\hline 15 & $\begin{array}{l}\text { Polymeric } \\
\text { Hydrogels }\end{array}$ & $\begin{array}{l}\text { Filomicelles \& } \\
\text { block co-poly- } \\
\text { mers (PEG-bl- } \\
\text { PPS) }\end{array}$ & $\begin{array}{l}\text { - Conformational change in } \\
\text { response to physiological or } \\
\text { photo-oxidation }\end{array}$ & $\begin{array}{l}\text { - At target site, Immunomodulatory NC-drug com- } \\
\text { plex self-assembles } \\
\text {-Sustained release for up to } 1 \text { month }\end{array}$ & 48 \\
\hline 16 & $\begin{array}{l}\text { Static Tissue Re- } \\
\text { generation Scaf- } \\
\text { folds }\end{array}$ & $\begin{array}{l}\text { porous, fibrous, } \\
\text { acellular, hy- } \\
\text { drogel, micro- } \\
\text { sphere, polymer }\end{array}$ & $\begin{array}{l}\text { - Facilitate the growth of tissue } \\
\text { cells for nearly any organ } \\
\text { - Permeable to cells, growth } \\
\text { factors, and biomolecules }\end{array}$ & $\begin{array}{l}\text { - Artificially stimulated cell proliferation in organ or } \\
\text { artificial implant therapies }\end{array}$ & 49,50 \\
\hline 17 & $\begin{array}{l}\text { Dynamic Tissue } \\
\text { Regeneration } \\
\text { Scaffolds }\end{array}$ & $\begin{array}{l}\text { Natural-PNI- } \\
\text { PAM polymer }\end{array}$ & $\begin{array}{l}\text { - Thermo-responsive } \\
\text { conformational changes } \\
\text { - Heat }>32^{\circ} \mathrm{C} \text { forms scaffold }\end{array}$ & $\begin{array}{l}\text { - Heat }<32^{\circ} \mathrm{C} \text { shrinks scaffold and releases tissue } \\
\text { culture } \\
\text { - Future, grow implantable tissue cultures in vitro } \\
\text { with patients cells }\end{array}$ & 51 \\
\hline 18 & $\begin{array}{l}\text { Thermosensitive } \\
\text { Nanogels }\end{array}$ & PNIPAM & $\begin{array}{l}\text { - Thermo-responsive } \\
\text { conformational changes }\end{array}$ & - Controlled drug release via NIR irradiation & 20 \\
\hline$* * *$ & stimuli-responsive & & & & \\
\hline
\end{tabular}


cellular plasmid delivery to mesenchymal stem cells (MSCs). This delivery mechanism significantly enhanced the transgene expression of bone morphogenetic protein-2 and vascular endothelial growth factor to stimulate MSC-mediated tissue regeneration (24). The star-PLLs are cationically charged, which facilitates a simple self-assembly synthesis procedure that facilitates strong adhesion of plasmid DNA (24). This NC also presents ideal properties to act as a framework for the gene therapy of various target cells in future applications. Another example of a self-assembled polymeric NC can be seen in a Diosgenin pro-drug anti-thrombosis agent where one molecule of the pro-drug is bound to a single PEG chain via a cleavable Schiff bass bond (25). When these monomeric units are placed in solution they self-assemble into PEG micelles that confer enhanced circulation time and controlled release of the prodrug in response to the acidic $\mathrm{pH}$ encountered upon arrival to the thrombus site (25). This prodrug facilitates the prevention of arterial and venous thrombus formation by increasing the subsequent Diosgenin therapies bioavailability and attraction to the desired endogenous targets (25).

Another vital group of polymeric NCs are those that are functionalized or composed of the naturally-derived polysaccharide, chitosan. Chitosan possess many ideal properties that make it therapeutically beneficial; it is biocompatible, biodegradable, mucoadhesive, antimicrobial, non-immunogenic, neuroprotective, and non-toxic (26-28). Additionally, the cationic surface charge of chitosan has been exploited in developing gene therapies to generate strong electrostatic interactions with gene silencing plasmids that facilitate stable delivery to the target site (27). Pure chitosan NPs express poor transfection rates, but modifications, such as complexation with polymers or lipids, have been shown to increase this ability (27, 28). For example, a genetically-targeted microbicide has been developed that is composed of lipids coated with a layer of chitosan that contains anti-HIV/SHIV siRNA-encoding plasmids (26). Based on the advantageous properties of chitosan, this NP was able to perform anti-HIV gene delivery to the intravaginal mucosal membrane (26). Chitosan has also been thiolated to further enhance the gene and drug delivering capabilities of the NPs. Thiol groups decrease the cationic surface charge of chitosan but does not interfere with the complexation of nucleic acids (28). In fact, this decrease in charge facilitates enhanced mucoadhesiveness, sustained drug delivery, and improved epithelial permeability $(27,28)$. In one study, a thiolated chitosan NC was utilized and shown to significantly enhance the anti-inflammatory effects of the anti-asthma drug, theophylline, due to the increased and prolonged absorption into bronchial epithelial cells (27). This NC presents an optimal vehicle for the treatment of cases of chronic allergic inflammation (27).

Another prominent group of polymeric NCs are lipid micelles, which have been being utilized to safely deliver toxic anti-cancer drugs to tumors. One example can be seen in the encapsulation and delivery of paclitaxel via micelles composed of a chitosan derivative, $\mathrm{N}$-succinyl-chitosan, and lipoic acid (29), In addition, these micelles were bound with low-density lipoprotein complexed with siRNA targeting multi-drug resistant pathways in cancer cells (29). This NC presents acidic -stimuli responsive behavior that induces drug release at the tumor microenvironment (29). Based on the enhanced tumor cell cytotoxicity, this NC presents a promising model for the co-delivery of siRNA and anti-tumor drugs to eliminate multi-drug resistant cancer cells (29). Another example can be seen in the treatment of lung cancer via DOX encapsulated in lipid micelles (30). However, this NC is subsequently loaded within Sertoli cells, which are biocompatible, naturally-derived cells that facilitate immunoprotection during drug delivery (30). Sertoli cells also avoid the physiological rejection of encapsulated NCs, and naturally translocate, become entrapped, and lyse within precapillary vascular beds within the lung (30). The Lysis facilitates the release of the lipid micelles and has been shown to increase the anti-tumor effects of DOX due to the enhanced and stable delivery (30).

Liposomal NCs are among the few that have been FDA approved for clinical application based upon their glorified amphiphilicity that facilitates non-toxic delivery of nearly any anti-cancer drug, and for their biocompatibility that allows them to be safely excreted and degraded within the body. Recently, a novel type of PEGylated liposomes was developed, called immunoliposomes (ILs), that are revolutionizing cancer immunotherapies. ILs can safely establish anti-cancer immunity by delivering cytokines and immune agonist antibodies that often cause severe toxicity due to high systemic exposure (31). In response, PEGylated immunoliposomes (ILs) are being developed that can establish anti-cancer immunity in a non-toxic manner. In one study, ILs had the immune agonists IL-2 and anti-CD137 covalently adhered to their surface and were functionalized to express significantly enhanced permeability and retention (EPR) in order to permeate into the tumor microenvironment (31). The ILs alter the cytokines' pharmacokinetics and biodistribution in a manner that successfully confers cancer immunity without generating toxic-effects (31).

However, a major challenge to conventional systemic drug delivery is the non-specific uptake of nanomedicines by the reticuloendothelial system (RES), which inhibits payload delivery and imparts toxicity within RES-associated organs, such as the liver, spleen, and kidney (32). In response, there has been a focus on developing nanoprimers that can prepare a patient's body for the subsequent administration of nanomedicines in a manner that will reduce the therapy's risk profile (33). In a recent study, it was demonstrated that the intravenous administration of intralipids one hour prior to therapy was shown to decrease RES uptake, while increasing the nanomedicine's bioavailability (32). Intralipids interact with the RES, which allows an increased amount of the nanomedicine to arrive at the target site (32). This study found these synergistic effects to exist for anti-cancer therapies using Abraxane, Marqibo, Onivyde, and a novel platinum-containing anti-cancer nanodrug. Advantageously, it is not required that modifications be applied to the payload or its carrier in order 
to confer this synergism, which makes it translatable to other BNT-based therapies (32). Another similar study utilized a non-toxic liposomal nanoprimer that presented low levels of hepatic accumulation and increased the subsequently administered nanomedicine's systemic bioavailability (33). It was found that by loading cytochrome inhibitor within the nanoprimer, the efficiency of docetaxel treatment significantly increased (33). In both cases, these lipid-based nanoprimers can be modified, through unique functional design, to express efficient priming for a broad range of nano-based therapies $(32,33)$.

Another novel liposomal application has been developed that utilizes liposome-nanobubble ultrasonic-responsive NCs for the enhancement of anti-cancer drug delivery (34). The LNs are comprised of phosphatidylserine and a paclitaxel payload, which confers pro-apoptotic effects that advance cancer cell death (34). In addition, the use of ultrasonic frequencies allows contrast-enhanced imaging of the location and tracking of the LNs in vivo (34). This allows them to be directly targeted with $12 \mathrm{MHz}$ frequency standard B-mode sonography to induce cavitation in the structure and enhance tumor-permeability of the LN (34). This ultrasonic-induced conformational change stimulates the on-demand release of paclitaxel, and through repeated exposure to the stimulus it was found that a controlled pulsated release was able to be accomplished. The conformation change also enhances the cellular internalization of the LN 10-fold, and the expression of paclitaxel-induced anti-cancer efficacy in vitro and in vivo (34).

Novel NCs are constantly in demand, and through vigorous BNT research, many have been discovered and are currently under investigation for their potential to enhance the delivery of conventional payloads or novel nanomedicines. A very promising recently discovered group of NCs are exosomes, which are endogenous cell-derived phospholipid vesicles ranging from 30-150nm in size that express biocompatible surface chemistry to their originator cells (35). Due to their biomimetic membranes, they are being examined for their function as enhanced targeted NCs that can deliver drugs across biological membranes and are non-immunogenic due to their biocompatibility with cell membranes. Exosomes have high loading capacities and can be greatly modified through surface conjugates to derive unique functionality, such as site-directed payload delivery and release (35). Another interesting function of exosomes can be seen in their utilization in the development of proteomic libraries by comprehensively analyzing the composition of exosomes that have budded from cancer cells $(36,37)$. Exosomes express the same surface biomarkers and internal molecular counterparts that are expressed on their originator cell, which provides information regarding the intracellular communication and signaling between cancer cells and potential therapeutic targets that can be utilized to enhance cancer therapy $(36,37)$. This technique has successfully identified over 6,000 cancer-associated proteins, many bioactive-cancerous agents, and various biomarkers that can be integrated into anti-cancer therapeutics (36).
Currently, techniques are being researched that can stimulate exosome biogenesis in a manner that produces consistently sized exosomes that can be utilized for reproducible and precise drug development (35). Endogenously, the biogenesis of exosomes is involved in cellular communication and chemical signaling $(35,38)$. The creation of reference databases that focus on the contents, functions, locations, and stimulants of these exosomes has stimulated research focused on enhancing the rate of biogenesis and increasing their bioavailability within diseased regions. For example, the stimulation of plasma-based exosomes was induced to deliver miR-24 intracellularly, which stimulates remote ischemic preconditioning that has the potential to attenuate reperfusion and myocardial ischemia-based injuries that could lead to stroke (38). miR-24 is endogenously produced and utilized in natural exosome communication and was found to be at highest concentration within plasma-based exosomes in rats (38). Induction of these exosomes enhances the delivery of miR-24, which reduces oxidative stress and cardiomyocyte apoptotic levels by downregulating the pro-apoptotic protein Bim (38). Unfortunately, the true mechanism of the induction of these exosomes is not well-established, but through this study important anti-ischemic molecular targets and endogenous anti-ischemic agents have been identified (38). As the exosomal induction mechanism becomes known, it will be able to be exploited, either genetically or though molecular induction, so that miR-24 can be more efficiently utilized for cardiovascular therapy (38).

\section{Inorganic NCs}

Currently, there are also large quantities of inorganic NCs in R\&D for their ability to enhance drug delivery systems. For example, mesoporous silica 21 NCs are being developed for their tunable pore size, high thermal conductance, avoidance of toxicity due to biocompatibility and targeted delivery, and high loading capacities $(39,40)$. In one study, MS NCs efficiently inhibited parasitic growth by safely delivering hydrophobic antimalarial drugs to targeted plasmodium infected red blood cells (39). In another study, antibodies were covalently bound to MS NCs in order to confer targeting focused on antigens specifically presented on the surface of leukemic stem cells (40). Through antibody-antigen binding reactions and proteomics studies, it was identified that IL-3 receptors are actively internalized upon antibody binding, presenting an optimal biomarker for highly effective targeted delivery of anti-leukemic medicines (40).

Gold NPs (AuNPs) are a conventionally popular NC due to their ideal physiochemical properties, but recent advancements in AuNP technology found that when they are bound with biocompatible polymer conjugates they can facilitate enhanced therapy for malignant tumors $(41,42)$. AuNPs have been shown to efficiently and safely deliver conventionally "difficult-to-deliver," hydrophobic and extremely toxic anti-cancer drugs, such as Doxorubicin (DOX) and bleomycin (BLM) $(41,42)$. The addition of polymers increases the AuNPs' stability, loading capacity, and cellular uptake (42). 
In a recent study focusing on DOX lung cancer therapy, it was found that DOX's ability to inhibit the growth of lung cancer cells was significantly enhanced by loading it within AuNPs that had polyvinylpyrrolidone polymers conjugated to the surface (42). This non-toxic nanomedicine facilitated highly efficient cellular internalization and intercellular release of DOX within lung cancer cells, which resulted in increased reactive oxygen species generation, sensitization of the cells, mitochondrial membrane potential, and induction of early and late apoptosis (42). In another recent study, it was demonstrated that PEGylated AuNPs can successfully facilitate non-toxic intracellular co-delivery of DOX and Bleomycin (BLM) to HeLa cells (41). The large difference in the mechanistic action of DOX and BLM presents a model that has the potential to decrease cancer drug resistance development while improving treatment efficiency (41). As this delivery system advances it is projected that it will be able to efficiently perform combination therapy focusing on ovarian cancer (41).

Another developing group of inorganic NCs are metal organic frameworks (MOFs), which are porous nanostructures that are constructed of metallic nanoparticles that are connected through organic linker molecules (43). MOFs express functional and structural tenability along with a high degree of porosity, which makes them applicable to drug delivery, catalysis, and sensing applications (43). In a recent study, aluminum-based MOF nanofactories were designed to protectively cage anti-oxidative superoxide dismutase and catalase and be delivered intracellularly to protect human cells from severe oxidative stress (43). These nanofactories were found to localize within the cell's lysosomes, but were still able to provide highly efficient enzymatic delivery for up to a week based upon their high chemical stability within low $\mathrm{pH}$ and significant protease resistance (43).

Zeolitic imidazolate frameworks (ZIFs) are another MOF that have recently been developed as a stimulus-responsive NC that can undergo conformational changes in response to alterations in environmental $\mathrm{pH}$ and interactions with biomimetic cell membrane particles, such as liposomes (44). This allows ZIFs to avoid premature drug release and nonspecific cellular interactions, and also confers the EPR effect when targeting cancer cells (44). ZIFs express a high degree of biocompatibility and structurally provide large, available pores that facilitate high drug loading capabilities (44). ZIFs are being examined for their efficiency in disease therapies associated with low $\mathrm{pH}$ environments, such as lysosomal and tumor microenvironments (44). For example, ZIF pH-responsive delivery of curcumin improved its bioavailability within tumors and its overall therapeutic effect (44).

\section{Nano-Platforms for Medical Applications}

During the last decades, advances in nano-engineered biomaterials such as hydrogels and nano-matrices have played a pivotal role in the development of controlled drug delivery systems. Drug release from these biomaterials is typically stimulated by an external stimulus, such as an applied magnetic field or photothermal energy. A major focus of such nano-biomaterials is the improvement of ocular drug delivery, because conventional ocular administration techniques typically waste nearly $95 \%$ of the drug $(45,46)$. Currently, an ocular nanowafer is being developed which is synthesized of a polymeric hydrogel embedded with a drug that slowly dissolves causing the gradual release of the drug $(45,46)$. Dentistry is another area that has greatly advanced based upon NBT advancements. Nano-polymeric dental adhesive resins are now being used as self-cleaning and self-healing restorative methods (3). Microneedle $(\mathrm{MN})$ patches are another drug delivery platform shown to be a painless and non-invasive alternative for transdermal administration (47). Recently, an alginate-based MN patch has been developed that has the potential to provide a safe, long-term and on-demand therapy for type 2 diabetes. The MN patch is integrated with Ex4 and glucose oxidase, which facilitates the glucose-responsive controlled release of exendin-4 (47).

A recent publication exemplified a filomicelle (FM) self-assembling scaffold made up of block copolymers (PEGbl-PPS) that provides non-inflammatory bioresorption and a high degree of customization (48). This allows it to be functionalized to perform sustained release of monodisperse micellar NCs for up to one month when incorporated in a hydrogel scaffold (48). This technology is being focused on immunomodulatory treatments for cancer, cardiovascular disease, and diabetes (48). The FM scaffold undergoes cylinder-to-sphere conformational changes when exposed to photo-oxidation or physiological oxidation due to changes in surface tension caused by the conversion of propylene sulfide into more hydrophilic derivatives (48). The speed of release can increase upon incorporation of photo-oxidizers, such as ethyl eosin, within the FM (48). However, the true novelty of this drug delivery mechanism stems from the retention and transport of non-loaded therapeutics, and the self-assembling of the NC-therapeutic complex at the target site (48). This was proven to be successful when analysis of the resulting spherical micellar NCs found them to be loaded with molecular payloads that were internally added to the FM scaffold (48).

In tissue regeneration, NT can be applied to reproduce or repair damaged tissues. By using suitable NM-based scaffolds and growth factors for artificially stimulated cell proliferation in organ transplants or artificial implants therapy, nanotechnology can be useful and potentially lead to life extension. Tissue regeneration is becoming a likely possibility as research works towards the development of scaffolds that facilitate tissue growth and are made from biocompatible NMs that are either natural compounds, such as collagen or chitosan, or synthetic compounds, such as PCL or PEG (49). There is a large variety of scaffolds currently in $\mathrm{R} \& \mathrm{D}$ that offer unique properties such as oxygen or growth factor delivery, but universally they all provide excellent tensile strength 49,50 . These factors facilitate the regeneration of a wide variety of tissues at various locations throughout the body $(49,50)$. Currently, these scaf- 
folds can be placed on or within a wound to stimulate smallscale tissue regeneration, which is enhanced by the scaffolds permeability to cells and biomolecules (49). In addition to static scaffolds there are dynamic scaffolds in R\&D that utilize thermo-responsive shape changing polymers, called PNIPAM (51). PNIPAM changes confirmation upon heating or cooling beyond the transition temperature of $32^{\circ} \mathrm{C}(51)$. PNIPAM polymers expand when heated and can be placed in molds that facilitate the formation of precisely scaled $3 \mathrm{D}$ tissue cultures (51). Once in the mold, the permeable PNIPAM scaffold can be inoculated with the desired tissue cells to produce the building blocks for tissue regeneration (51). The tissue culture can be released by reducing the temperature and can be utilized for in vitro drug response profiling (51). As this technology progresses these building blocks will facilitate the complete growth of tissue culture derived organs (51). Additionally, since the organ would be derived from the patient's cells, it is expected that there would be a lack of rejection complications during transplantation.

In addition, PNIPAM has begun to be studied for its application towards facilitating non-invasive stimuli-responsive drug release. Currently, PNIPAM nanogels have been successful in controlling drug release in vitro, which is advantageous regarding the implications towards assay development (20). The thermosensitive responsiveness of PNIPAM facilitates the generation of specifically drug release profiles through the administration of controlled irradiation via NIR light (20). These nanogels were loaded with chitosan-modified chemically reduced graphene oxide vehicles that contained DOX (20). Upon irradiation this platform was able to release a cytotoxic level of DOX in a controlled manner, which shows promise for future in vivo applications (20).

\section{Nano-Theranostics (Table 2)}

Nano-theranostics are nanoformulations that simultaneously deliver a payload and an imaging agent, such as a contrast agent or luminescent molecular tag, to a targeted location in order to confer therapeutic benefit, track bioactivity during administration, decrease toxicity of conventional imaging agents, and enhance the capabilities of nano-imaging instruments. By incorporating targeting moieties onto a theranostic nanoformulation, site directed imaging can be significantly enhanced, which aids in disease detection capabilities. For example, a theranostic nanoformulation is being developed that can target pancreatic cancer cells for the delivery of gemcitabine, an anticancer drug, and for labeling with gadolimium, a potent MRI contrast agent $(52,53)$. The major advantage is that both of these compounds are toxic in their free form and can cause many complications, but in this case, toxicity is eliminated $(52,53)$. The gadolinimum allows MRI tracking of the nanoformulation during systemic circulation, ensuring delivery to the target location is successful $(52,53)$.

However, manganese $(\mathrm{Mn})$ has recently been replacing gadolinium as a less-toxic T1 MRI contrast agent. Mn also expresses paramagnetic properties and an extended plasma half-life that greatly enhance the targeting capabilities for theranostic delivery (54). For example, a PEGylated lipid micelle, DOPE, DC-Cholesterol, with a Mn-oxide core was developed for the co-delivery of siRNA-encoding plasmid DNA and DOX for the treatment of lung cancer (54). The formulation was administered intranasally to mice and was found to provide an efficient vehicle for cancer lung theranostics based upon the significant anti-cancer effect, excellent T1 MRI contrast, and enhanced accumulation within the lungs (54).

Another theranostic approach currently being developed targets the endothelial cells of inflammatory sites using novel biomimetic NCs called leukosomes (55). Leukosomes have properties similar to liposomes regarding their loading capacities, ability to be functionalized, and biocompatible composition due to their endogenous nature (55). In this study, the leukosomes were targeted to endothelial biomarkers, VCAM-1 and ICAM1 (55). These markers also show promise as potential targets for early diagnosis of atherosclerotic conditions (55). The leukosomes proved to be efficient vehicles for delivery of a broad range of anti-inflammatory agents, as well as the co-delivery of MRI contrast agents (55).

Chitosan has also been utilized to functionalize many NPs so that they can perform theranostic functions that focus on delivering therapeutic plasmids and magnetic resonance imaging (MRI) contrast agents that allow the researchers to treat and visualize brains of individuals suffering from traumatic brain injury or other neurodegenerative diseases. A recently developed chitosan NP has been developed that targets the brain and incorporates manganese as a T1 MRI contrast agent (56). The manganese is contained within a cross-linking agent, Mangafodipir, that forms a stable chitosan-plasmid DNA matrix (56). Upon intranasal administration, this NP was found to efficiently translocate to the brain and is hypothesized to be endosomally internalized by olfactory sensory neurons (56). The chitosan matrix then becomes degraded by the endosomal environment, which releases the plasmids intracellularly so that they can perform gene silencing (56). Another MRI contrast agent that has been complexed with chitosan is superparamagnetic iron oxide nanoparticles (SPIONs), which also facilitate magnetofection. Magnetofection is the capability to attract NPs to a specific area of the body through the application of an external magnetic field (57). An example is SPION-containing block-polymer micelles coated with cationic polyethyleneimine (PEI) and chitosan complexed with DNA $(57,58)$. These micelles were able to magnetically translocate to the brain, efficiently release the plasmid DNA, and facilitate enhanced in vivo imaging $(57,58)$.

Additionally, chitosan has been complexed with graphene oxide to generate optimal non-toxic theranostic gene delivery vehicles that are showing promise for their applicability towards gene, chemo- and phototherapies focused on the treatment of cancer (59). The major advantage of these chitosan-graphene oxide NPs is that they can facilitate the co-delivery of anti-cancer plasmid DNA, DOX, and MRI contrast agents (59). This combinatory approach has generated a non-toxic and non-invasive platform for cancer therapy and in vivo tumor imaging and diagnostics (59). 
AuNPs have also been utilized for theranostic therapies targeted to inflammatory sites that correspond to atherosclerosis (60). AuNPs of various sizes and shapes are being utilized for their ability to passively and actively target inflammatory sites based upon affinity to specific inflammatory biomarkers, such as accumulation of monocytes and macrophages in high risk plaques (60). Upon arrival, the AuNPs act as a contrast agent because they can become stimulated with photoacoustic (PA) waves emitted from a PA tomography laser (60). This produces detectable pressure acoustic waves that are induced by the thermoelastic expansion of the AuNPs (60). AuNPs also showed the ability to target sites of thrombosis through biomarkers such as the cell surface glycoprotein receptor Integrin $\alpha \mathrm{V} \beta 3$, which is expressed on activated endothelial cells and can be utilized to visualize CVD-related angiogenesis (60). In this study AuNPs were also presented for their capability, in cohort with PA stimulation, to induce the opening of the blood brain barrier in rats when exposed to focused-ultrasound (60). This ability would allow researchers to better diagnose and study the molecular environment of CVD-induced brain-related symptoms, such as ischemic strokes (60). Overall, AuNPs are showing great promise in regard to their ability to function as a contrast agent, and also present theranostic potential due to their large and flexible loading capacities that can be utilized for the delivery of a broad range of nanomedicines (60).

Table 2. Novel Nano-Theranostics (Section 3.0)

\section{Theranostic Nanocarriers (Section 3.0)}

\begin{tabular}{|c|c|c|c|c|c|c|}
\hline \# & Type & Material & \multicolumn{2}{|l|}{ Novel Physiochemical Properties } & Novel Theranostic Applications & Ref \\
\hline 1 & Liposomes & $\begin{array}{l}\text { Phospholipid } \\
\text { bilayer }\end{array}$ & \multicolumn{2}{|c|}{$\begin{array}{l}\text { - Bypass toxicity of potent anti-cancer } \\
\text { and MRI contrast agent }\end{array}$} & $\begin{array}{l}\text { - Efficient pancreatic cancer therapy } \\
\text { - Systemic MRI tracking }\end{array}$ & 52,53 \\
\hline 2 & Lipid Micelle & $\begin{array}{l}\text { DOPE, DC-Choles- } \\
\text { terol }\end{array}$ & \multicolumn{2}{|l|}{$\begin{array}{l}\text {-Co-delivery of DNA, DOX, and man- } \\
\text { ganese }\end{array}$} & $\begin{array}{l}\text {-Enhanced accumulation in lungs, } \\
\text { Ideal for lung cancer theranostics }\end{array}$ & 54 \\
\hline 3 & Leukosome & $\begin{array}{l}\text { Biomimetic lipid } \\
\text { Bilayer }\end{array}$ & \multicolumn{2}{|l|}{$\begin{array}{l}\text { - Functionalized surface with athero- } \\
\text { sclerotic biomarkers for enhanced } \\
\text { targeted co-delivery }\end{array}$} & $\begin{array}{l}\text { - Track delivery of anti-inflammatory } \\
\text { agents to atherosclerotic plaques } \\
\text { - Early diagnosis of conditions }\end{array}$ & 55 \\
\hline 4 & Micelles & $\begin{array}{l}\text { Block copolymers } \\
\text { w/ chitosan }\end{array}$ & \multicolumn{2}{|l|}{$\begin{array}{l}\text { - Biodegradable chitosan matrix } \\
\text { protects and controls release of its } \\
\text { payload }\end{array}$} & $\begin{array}{l}\text { - Magnetofection driven gene therapy } \\
\text { - efficient bypassing of the BBB }\end{array}$ & $56-58$ \\
\hline 5 & AuNPs & $\mathrm{Au}$ & \multicolumn{2}{|l|}{$\begin{array}{l}\text { - AuNPs are PA contrast agents, } \\
\text { induction causes thermoelastic ex- } \\
\text { pansion }\end{array}$} & $\begin{array}{l}\text { - Diagnose of cardiovascular related heart } \\
\text { and brain symptomatic profile } \\
\text { - Ultrasonic induced opening of BBB }\end{array}$ & 60 \\
\hline \multicolumn{7}{|c|}{ Novel Theranostic Tumor Ablation Therapies (Section 3.1) } \\
\hline \# & \multicolumn{2}{|c|}{ Nano-Theranostic Formulation } & Ablation Therapy \& Stimulus & & Anti-Cancer Activity & \\
\hline 6 & \multicolumn{2}{|c|}{$\begin{array}{l}\text { NCs } w / \text { radiosensitive metal na- } \\
\text { noenhancers }\end{array}$} & $\begin{array}{l}\text { - Radiotherapy, stimulated via } \\
\text { gamma or } \mathrm{X} \text { rays }\end{array}$ & \multicolumn{2}{|c|}{$\begin{array}{l}\text { - Oxidative stress, cell cycle arrest, or inhibit } \\
\text { DNA repair }\end{array}$} & 61 \\
\hline 7 & \multicolumn{2}{|c|}{$\begin{array}{l}\text { NCs w/ magnetic superparamag- } \\
\text { netic Iron Oxide NPs }\end{array}$} & $\begin{array}{l}\text { - Hyperthermic, stimulated via } \\
\text { exposure to alternating magnetic } \\
\text { field }\end{array}$ & \multicolumn{2}{|r|}{$\begin{array}{l}\text { - High heat produced by quick alternation fre- } \\
\text { quency of the magnetic NPs magnetic moments }\end{array}$} & 62,63 \\
\hline 6 & \multicolumn{2}{|c|}{$\begin{array}{l}\text { Photosensitive melanin loaded } \\
\text { PEGylated liposomes }\end{array}$} & $\begin{array}{l}\text { - Photothermal, stimulated via } \\
\text { exposure to } 808 \mathrm{~nm} \text { NIR light }\end{array}$ & \multicolumn{2}{|c|}{$\begin{array}{l}\text { - In vivo photoacoustic imaging \& MRI guided } \\
\text { photothermal ablation of tumors in breast can- } \\
\text { cer patients }\end{array}$} & 64 \\
\hline 7 & \multicolumn{2}{|c|}{$\begin{array}{l}\text { Lipid membrane coated Hollow } \\
\text { Silica-Carbon NPs loaded w/ pho- } \\
\text { tosensitizing agents }\end{array}$} & $\begin{array}{l}\text { - Photodynamic, stimulated via } \\
\text { NIR exposure \&subsequently via } \\
\text { interaction w/ ROS }\end{array}$ & \multicolumn{2}{|c|}{$\begin{array}{l}\text { - Increases (NAD+), decreases (ATP) available for } \\
\text { ATP-efflux pumps, blocks multi-drug resistant } \\
\text { properties in cancer cells for } 5 \text { days } \\
\text { - Creates window for treatment }\end{array}$} & 66 \\
\hline \multicolumn{7}{|c|}{ Surgical Applications of Theranostics (Section 3.2) } \\
\hline \# & \multirow{2}{*}{\multicolumn{2}{|c|}{ In vivo Fluorescent Guided Therapy }} & $\begin{array}{l}\text { Nano-Theranostic } \\
\text { Formulation }\end{array}$ & & Advantage to Precision Surgery & \\
\hline 9 & & & $\begin{array}{l}\text { - NCs w/ specific fluorescently } \\
\text { tagged cancer biomarkers }\end{array}$ & $\begin{array}{l}\text { - Ev } \\
\text { site }\end{array}$ & $\begin{array}{l}\text { ery, and only, cancerous cell in a surgical } \\
\text { is illuminated to ensure complete removal }\end{array}$ & $69-71$ \\
\hline 10 & \multicolumn{2}{|c|}{$\begin{array}{l}\text { NIR Fluorescence-Enhanced Diffuse } \\
\text { Optical Tomography - multimodal } \\
\text { use of real-time computed tomog- } \\
\text { raphy \& MRI }\end{array}$} & $\begin{array}{l}\text { - Tumor targeted NCs incorporat- } \\
\text { ing fluorescent NPs }\end{array}$ & \multicolumn{2}{|r|}{$\begin{array}{l}\text { - Real-time temporal and spatial 3D imaging for } \\
\text { the identification of biological \& genetic causal- } \\
\text { ities of disease } \\
\text { - Earlier detection \& removal of tumors }\end{array}$} & $72-73$ \\
\hline
\end{tabular}




\section{Theranostics for Tumor Ablation in Cancer Patients}

Stimuli-responsive nanoparticles are being incorporated into nanoformulations that are targeted for tumor cells, and upon radiation, photonic, thermal, or magnetic stimulation they can induce complete cancer cell ablation and enhanced tumor theranostics and avoid cytotoxic effects on healthy cells that have conventionally been an issue regarding patient safety. Radiotherapy, for instance, is a very effective mechanism for the treatment of cancer but has typically been linked to severe adverse effects due to the toxicity of the radiosensitizing agents administered to the patient (61). However, safe and highly efficient radiotherapy can be accomplished in vivo through the utilization of biocompatible tumor-targeted NCs incorporated with chemically inert radiosensitive metal-based nano-enhancers, such as gold, tungsten sulfide, iron, silica, and gadolinium (61). Once at the tumor sites they can be stimulated through gamma-rays and X-rays, which results in cancer cell death through the induction of oxidative stress, mitochondrial failure, cell cycle arrest, or inhibition of DNA repair (61).

Magnetic NPs, such as superparamagnetic iron oxide, are another additive agent that has shown great promise towards the advancement of non-invasive and safe cancer therapy through targeted magnetically-stimulated ablation of tumor cells, which is referred to as hyperthermia $(62,63)$. A recent study found that hyperthermic efficiency was affected by the shape of the iron oxide NPs, with square shapes generating more heat energy and able to be synthesized at smaller sizes than spheres (63). An extremely quick alternating external magnetic field is applied to magnetic NPs that have arrived at the tumor site, which causes their magnetic moments to alternate at a frequency that generates enough heat to ablate the cancer cells (62). Magnetic NPs also confer theranostic capabilities because most act as either T1 or T2 contrast agents for MRI (63). This ability to image the NPs in vivo allows the tumor cells to be precisely located and targeted in a manner that avoids inducing cytotoxic effects towards healthy cells (63).

Photo-sensitive nanoparticles that absorb NIR light and convert it into heat are another optimal agent to incorporate into anti-cancer medicines to accomplish complete tumor ablation in response to destabilizing increases to the internal temperature of the cells (64). This is referred to as photothermal therapy (PTT) and has been proven to exemplify superior, non-invasive anti-cancer effects when compared to conventional surgical and chemotherapeutic techniques (64). Additionally, after PTT a patient experiences a short recovery period, significant decrease in the occurrence of toxic effects, and low cancer recurrence rate (64). A recent study discovered that endogenous melanin exemplifies optimal photothermal conversion efficiency to be utilized as a photo-sensitizing agent in PTT targeted with an 808nm NIR laser for 10 minutes (64). However, when administered alone, the PTT efficiency is not significant (64). In response, melanin was loaded within PEGylated liposomes, which mitigated melanin's potential toxicity and conferred theranostic capabilities of simultaneous in vivo photoacoustic imaging and MRI guided photothermal ablation of tumors (64). It was found that when administered in mice, complete tumor ablation was successful in mice infected with breast cancer (64). Based upon its bio-safe nature and high photothermal efficiency, this theranostic nanomedicine shows great promise for translation into clinical settings, and as a framework for future PTT development for other types of cancers.

Another application of photosensitizing agents can be seen in a study where hollow silica NPs were loaded with photosensitizing agents and surface-functionalized for enhanced retention due to charge stabilization within the acidic tumor environment, which allowed them to be the active agent for the induction of tumor ablation through non-invasive photodynamic therapy (PDT) (65). PDT is oxygen dependent, and upon photonic stimulation this PDT agent has been shown to induce the production of endogenous hydrogen peroxide, ROS, which facilitates cellular internalization and causes mitochondrial failure (65). Interestingly, in another study ROS generation was stimulated to block multi-drug resistance in cancer cells by altering the amount of ATP available for the overexpressed ATP-driven transmembrane efflux pumps (66). This was accomplished through the administration of lipid membrane-coated silica-carbon (LSC) hybrid NPs that were targeted to the pyruvate molecule found in the mitochondria (66). Upon NIR radiation, the generation of ROS begins, resulting in a larger concentration of NAD+ through oxidation of $\mathrm{NADH}$ (66). This example of PDT destroys the multi-drug resistance for up to five days, which provides a therapeutic window for the treatment of multi-drug resistant bacteria (66). This therapy could generate a framework to address the growing challenge and serious risk that is presented by the potential emergence of multi-drug resistant cancer cells.

The final type of stimuli-responsive cancer therapy that is currently rising to popularity is facilitated by redox-responsive NCs. These NCs are responsive to the change in glutathione (GSH) concentrations as they internalize into cells, which have a much higher concentration in comparison to the extracellular environment (67). The gradient the NCs are exposed to in terms of reduction potential is being harnessed as an endogenous stimulus that can trigger cytosolic intracellular release (67). This mechanism has been especially applicable to cancer therapies due to the presence of a GSH concentration in tumor cells that is four times greater than that of healthy cells (67). For example, echogenic polymersome vesicles are a novel theranostic redox-responsive drug delivery system composed of extremely stable amphiphilic polymers, and encapsulated with air bubbles that facilitate diagnostic ultrasound imaging (68). These polymersomes were functionalized for folate-targeting, and the co-delivery of gemcitabine and DOX (68). Currently this model has only been tested within in vitro $3 \mathrm{D}$ spheroid cultures, but was shown to express enhanced uptake and GSH stimulated intracellular release within breast and pancreatic cancer cells (68). However, this model has the potential to be translated into an in vivo setting and confer potent anti-cancer effects. 


\section{Surgical Application of Theranostic Nanoparticles}

A major focus of BNT diagnostics is the development of cancer imaging techniques to identify and locate cancer cells. For example, an in vivo tumor imaging technology is being developed, called in vivo fluorescent guided therapy, which fluorescently illuminates every cancerous cell in the patient in order to ensure complete and precise surgical removal (69). This technique even illuminates cancer cells that have not yet formed into visible tumors, which reduces the risk of cancer recurrence after surgery (69). Cancer cells are targeted by specific biomarkers that are fluorescently tagging by theranostic NCs. The combination of diagnostic techniques and bioinformatics allows the most unique, specific biomarkers for each type of cancer to be identified for use as the target molecule, which ensures that only cancerous cells will be targeted. This technique has been successful in mouse models and is on its way to becoming a common technique for the enhancement of precision surgery (69-71).

Another unique diagnostic technology that has been developed utilizing multimodal in vivo imaging technology is called Optical Tomography, which performs multimodal imaging utilizing $\mu \mathrm{CT}$ and MRI to produce $3 \mathrm{D}$ optical images $(72,73)$. NIR fluorescence-enhanced diffuse optical tomography (fDOT) has already been used to localize tumors in mice using fluorescent nanoparticles. This technique utilizes engineered bioluminescence or fluorescent tagging to perform non-invasive in vivo longitudinal and real-time imaging and temporal and spatial defining of biological and genetic process associated with disease and drug delivery $(72,73)$. Such techniques will lead to better understanding and diagnostic capabilities to detect diseases at earlier stages based upon identification of issues within biological mechanisms and their specific causes $(72,73)$.

\section{DNA-based Biomedical Nanotechnology Applications}

(Table 3)

DNA technology, regarded as DNA origami (DNA-O), is currently rising to popularity due to its ability to perform a broad variety of functions, including drug delivery, in response to the flexibility of the design of the final structure. Additionally, synthesis and production of DNA-O components and structures is fairly economical and easily achieved with DNA amplification techniques, such as PCR, and programmable self-assembly through complementary sequence design (74). DNA technology has been greatly advanced by computational programs capable of facilitating precise design modeling that can be applied to actual production of these non-conventional DNA-O structures (75). These programs convey a high degree of control over the final structural characteristics, surface chemistry and functionality (74-76).

Primarily, DNA-O is being utilized to develop novel smart drug delivery systems based upon its biocompatible nature, amphiphilic structure, and ideal physiochemical properties for drug loading, delivery, and release. $(74,76)$. For example, DNA-O NCs are being examined for their enhancement towards the delivery of hydrophobic anti-cancer drugs, such as DOX (74-76). Also, the structural customization of DNA-O NCs allows them to express high affinity ligand-receptor interactions, and if desired, biomolecules can be incorporated that confer stimuli-responsive behavior that can be designed for nearly any stimulus (74-76). An example can be seen in the delivery of photosensitizing agents, such as gold nanorods, within targeted DNA-O NCs that can facilitate photothermal ablation of cancer cells (76). However, DNA-O NCs are not limited to cancer; they can be nearly ubiquitously adapted to efficiently treat any disease and deliver any drug. For instance, they are currently being integrated into the CRISPR/Cas9 genomic editing system in order to provide stable and precisely targeted delivery of the Cas9 proteins (76). Another unique advancement has been seen in the development of a DNA-O-based scaffold that is designed to match the curve of cellular membranes (77). This scaffold is being examined for its potential to repair damaged cellular membranes through binding and subsequent release of loaded biomolecules that facilitate cellular repair and potentially cell membrane genesis (77).

Table 3. DNA-based Biomedical Nanotechnology Applications (Section 4.0)

\section{DNA Origami - (DNA-O)}

$\#$ Type

1 Targeted DNA-O NCs

2 DNA-O Scaffold

3 DNA Nanorulers

4

DNA Integrated Live Cell Circuits
Novel Physiochemical Properties

- Stable \& targeted intracellular delivery of Cas9 proteins

- Delivery photosensitive gold nanorods to tumor cells

- Biomimetic curved-shape matches the curve of a cell membrane

- Nanoscale DNA strands of exact size w/ fluorescent tags at equal intervals

- DNA-based "memory storage" system that can accurately respond to extracellular signals through the production of a DNA strand that will encode the required response
Novel Therapeutic Outcome

- Enhanced CRISPR/Cas9 genomic editing

- Vehicle to facilitate photothermal ablation

- Repair of damaged cell membranes by delivering essential biomolecules

- Enhance the accuracy of nanoscale measurements in microscopy

- Diagnostic extracellular biomolecule detection

- Stimulate existing cellular communications that induce specific cell states - Future, rewire existing signaling pathways 
DNA-O can also provide NCs that can facilitate the safe delivery of contrast agents and fluorescent tags or markers to target sites to enhance in vivo imaging capabilities (74-76). A novel DNA-imaging agent is currently being developed, called DNA nanorulers, which provides a tool that can be delivered to sites destined for analysis by fluorescent microscopic imaging (78). These nanorulers are precisely synthesized to be an exact size, and also incorporate fluorescent tags at equal intervals along the DNA strand in a manner that simulates the ticks of a ruler (78). DNA nanorulers present the potential to significantly enhance the accuracy of nanoscale measurements of imaged biomolecules through superresolution (78).

Lastly, DNA-O exemplifies properties that make it advantageous for application towards the development of DNA/live cell complexes that simulate electronic circuitry. These are called integrated live-cell circuits, and they can develop appropriate responses to molecular or stimulus-based inputs (75). The input stimulates the circuit to access incorporated DNA-O memory storage structures that release the appropriate DNA strand that encodes the desired output (75). The released signals stimulate the cell's endogenous biosynthetic machinery to produce the desired DNA sequence that becomes either building blocks to repair the circuit or templates to produce outputs and cellular signals in the form of proteins or genetically encoded fluorescent reporters (75). These circuits can be used in diagnostic studies to analyze extracellular biomolecule presence, but can also be prospectively utilized to trigger intracellular cellular signaling pathways in a stimuli responsive and controlled manner (75). Eventually, the goal is to be able to implant precisely programmed DNA circuits into in vivo biological systems in order to stimulate existing cellular communications that induce specific cell states, such as apoptotic states to fight against detected disease inputs (75). Additionally, researchers are examining ways that these circuits can be programed in a manner that rewires existing signaling pathways to induce novel or enhanced functionalities (75).

\section{Nano-enabled Diagnostics}

BNT-enabled diagnostics has incorporated mechanical and electrical NTs to create bioanalytical devices called Biological Micro-Electro-Mechanical Systems (BioMEMS). Initially BioMEMS application was primarily in vitro to characterize physiochemical properties of nanoparticles such as size, mass, and zeta potential; detection of specific biomarkers for disease diagnostics; or identification of contaminants or pathogens in biological samples such as body fluids, or water samples $(79,80)$. A major advantage of BioMEMS is their ability to perform HTS at an extremely fast rate for many samples $(79,80)$. However, the miniaturization and advancements towards micro-cantilever technology has further increased the accuracy, sensitivity and detection capabilities of BioMEMS while decreasing the cost, the amount of solvent and sample used per assay, and the speed of result acquisition $(79,80)$. The continued trend of miniaturization has led to the generation of cell-on-a-chip technologies that have the capability to perform analysis on the level of single cells (81). Also, organ-on-a-chip technologies have been generated that simulate $3 \mathrm{D}$ tissue environments that represent in vivo cell-cell interfaces and can accurately depict biochemical, genetic, and metabolic activities in real time $(80,81)$. These biosensor-based chip technologies have made it possible to detect diseases in their earliest stages due to their extremely small detection limits and their ability to detect the presence of disease cell-by-cell $(80,81)$. These technologies will revolutionize in vitro assays and allow simple, non-invasive, inexpensive devices for patient-performed in-home diagnostics. A limiting factor off the chip technologies is that they only allow extracellular and surface-cellular detection, but another biosensing technology called nanoscale endoscopes allows subcellular detection of organelles and cytoplasmic biomarkers (82). The endoscope non-invasively probes a cell and photonically stimulates fluorescently tagged organelles to detect diseased cells based on individual intracellular signals (82). This technique provides insight into intracellular disease profiles, which will significantly aid in the development of future therapies.

Recently, molecular electrodes have been developed for diagnostic purposes. A promising model for the electrochemical detection of mutated DNA sequences and single-nucleotide polymorphisms utilizes graphene electrodes that are functionalized with chitosan (83). Graphene's physiochemical properties make it an excellent material for the generation of biosensors due to its exceptional electronic, mechanical, and thermal properties (83). The chitosan facilitates the covalent attachment and immobilization of a single stranded DNA sequence that is complementary to the target sequence (83). The electrode platform is added to the biosensing device, which is then subjected to voltammetry (83). During the assay completely, complementary DNA sequences bind the DNA of the electrodes. This determines if the target sequence is present in the biological sample (83). This rapid and inexpensive platform presents the potential to be utilized as a point-of-care diagnostic device that can detect disease-related DNA mutations in a sample taken from a patient (83). As this technology continues to progress multiplexed electrodes can be generated that will be able to characterize the genetic profile of an individual in comparison to a specific disease (83).

There are also many types of bioassays that are being developed that provide in vitro models that closely represent in vivo microenvironments and enhance the precision and accuracy of drug response profiling. For example, polymeric electrospun fiber-inspired smart scaffolds (FiSS) are being utilized during in vitro cancer studies to facilitate the formation of $3 \mathrm{D}$ tumoroids (84). The tumoroids are formed from extracted tumor cells of a specific cell line that were grown in mice (84). Advantageously, tumoroids exemplify reduced sensitivity towards anticancer drugs, which improves the quality of screenings for anticancer compounds in comparison to the classical models (84). Additionally, this technology can be utilized to culture tumor cells from patient biopsies to perform accurate diagnostics that can aid in the development of personalized cancer therapies (84). Another example can be seen in in vitro microfluidic 
blood brain barrier (BBB) models that accurately represent in vivo conditions (85). Additionally, single cell additive manufacturing is assisting in the generation of $3 \mathrm{D}$ bioprinted in vitro tissue and organ models that can be utilized in regenerative research (86).

Additionally, lipid technologies have begun to revolutionize high throughput in vitro assays by decreasing the size of each compartment (or well) on microarrays using evaporative edge lithography to stamp lipid bilayers in a specific orientation, producing microarrays with the potential to test 50,000 drug candidates in a single assay (87). This lipid technology was also used to develop lipid-oil droplet arrays that do not require additional solvent and can assay lipophilic drugs (87). These assays can also determine the dose-dependent bioactivity of large quantities of small molecules to determine their potential as a nanotherapeutic in nanomedicine $(88,89)$. This lipid technology has also been used in the development of "nanointaglio," which detects analytes in air vapors by optically sensing topographical changes to lipid grates upon the passage and binding of vapor analytes (88-90). Overall, bioMEMS are completely revolutionizing in vitro analyte and disease diagnostics, biological sample analysis, NP characterization, and lead selection through bioactivity high throughput screenings.

Another truly innovative technology is the development of nano-based implants to repair damaged or inactive sensory organs, such as an artificial ear that can allow a deaf person to hear or an artificial nose that can grant someone the sense of smell $(90,91)$. These implants are essentially BioMEMS that are specifically functionalized to detect sensory stimuli and use the electronic aspects to send signals to the desired cells, typically central nervous system cells $(90,91)$. For example, a cochlear ear implant is being developed that utilizes nanoelectrodes to plasmonically stimulate neural and cardiac cells to confer the ability to hear for the hearing-impaired (91). Nano-implantable technologies, such as gradually dissolving hydrogels, are also in development for implantable diagnostic devices and implantable drug administration devices that can allow point-of-care, in-home treatment and controlled drug release without issues of patient non-compliance. The future goals of BNT include innovative technologies with the potential to facilitate drastic changes in what was once believed to be irreversible damage and permanent health conditions of the human body, such as amputation or inert deafness. These goals are being accomplished through the combination of functionalized BioMEMS, "smart" biomaterials, and 3D bioprinting technologies.

\section{Future Prospects of Biomedical Nanotechnology}

In conclusion, the BNT revolution is proving to be a continually growing dynamic force that is generating innovations that are altering the world's perspective on medical care. Specifically, there will be a rise in the production and widespread utilization of highly therapeutic efficient personalized and precision nanomedicines and nanotherapies. This integration will facilitate the elimination of the fear of mortality from conventionally chronic or fatal diseases and is also projected to discover therapies that will extend the average lifespan. In cases where tumors or harmful growths develop precision and assisted surgical techniques are greatly advancing to the point where they can surgically remove single cells. Also, to repair surgical sites or any form of tissue damage, BNT is facilitating the generation of many platforms for tissue regeneration that utilize an individual's cells, which will lead to the elimination of tissue implant rejection. In the future the treatment burden to a patient will be significantly decreased based upon the development of prolonged and stable delivery systems that will lower the number of administrative events. Additionally, many nanomedicines will be formulated into non-invasive, self-administrable, and high-patient-compliant administrative forms that will further increase the ability for the patient to perform inhome therapy. Diagnostic devices, such as BioMEMS, will also continue to become more cost-effective and easily functional so that patients can perform in-home point-of-care diagnostics, which will also aid in the earlier detection of disease. As these BioMEMS integrate with computer technology they will be able to perform real-time communications with health care facilities upon identification of disease and serious compliance or toxicity issues. BioMEMS will also present products that will be able to function as in vivo implants acting as functional or sensory organs. Regarding scientific development, microscopy and characterization instrumentation will continue to increase in functionality, precision, and accuracy which will lead to particle level detection and differentiation. Overall, if implemented in accordance with appropriate regulation and safety-monitoring, BNT has the potential to enhance the worldwide quality of life for humans across every continent.

\section{Abbreviations}

BNT - Biomedical Nanotechnology, NT - Nanotechnology, NC - Nanocarrier, R\&D - Research and Development, FDA - Federal Drug Administration, HTS - High-Throughput Screening, BBB - BloodBrain Barrier, NP - Nanoparticle, NM - Nanomaterial, Star-PLL Star-Shaped Poly (L-lysine) Polypeptide, MS - Mesoporous Silica, AuNPs - Gold Nanoparticles, DOX - Doxorubicin, BLM - Bleomycin, MOF - Metal Organic Framework, EPR - Enhanced Permeability and Retention, ZIF - Zeolitic Imidazolate Framework, LN - Liposome Nanobubbles, MN - Microneedle, FM - Filomicelle, PEG, PCL, PNIPAM, PA - Photoacoustic, MRI - Magnetic Resonance Imaging, PTT - Photothermal Therapy, NIR - Near Infrared

\section{Acknowledgements}

The authors would like to acknowledge Ms. Christen Bouchard for assistance in preparation of the manuscript.

\section{Funding Information}

This research was supported by VA Merit Review Awards \#BX003685 and BX003413, and Research Career Scientist Awards to Dr. Shyam Mohapatra (IK6 BX003778) and Dr. Subhra Mohapatra (IK6BX004212). Though this report is based upon work supported in part by the Department of Veterans Affairs, Veterans Health Administration, Office of Research and Development, the contents of this report do not represent the views of the Department of Veterans Affairs or the United States Government. The Department of Veter- 
ans Affairs had no role in data collection and analysis, decision to publish, or preparation of the manuscript.

\section{Conflict of Interest Statement}

All authors have indicated no financial conflicts of interests in relation to writing and contents of this review. Both Subhra Mohapatra and Shyam Mohapatra are co-founders and act as scientific advisors in Transgenex Nanobiotech Inc., a University of South Florida (USF) Spin-out company. The terms of the conflict management have been reviewed and approved by the USF conflict of interest policies.

\section{References}

1. Iavicoli I, Leso V, Ricciardi W, Hodson LL, Hoover MD. Opportunities and challenges of nanotechnology in the green economy. Environ Health 2014; 13:78.

2. Hutchison JE. The Road to Sustainable Nanotechnology: Challenges, Progress and Opportunities. ACS Sustain Chem Eng 2016; 4:5907-5914.

3. Cheng HN, Doemeny LJ, Geraci CL, Grob Schmidt D. Nanotechnology Overview: Opportunities and Challenges. Nanotechnology: Delivering on the Promise Volume 1. Volume 1220: American Chemical Society, 2016:1-12.

4. Purohit R, Mittal A, Dalela S, Warudkar V, Purohit K, Purohit S. Social, Environmental and Ethical Impacts of Nanotechnology. Materials Today: Proceedings 2017; 4:5461-5467.

5. Di Sia P. Nanotechnology Among Innovation, Health and Risks. Procedia Soc Beh Sci 2017; 237:1076-1080.

6. Harifi T, Montazer M. Application of nanotechnology in sports clothing and flooring for enhanced sport activities, performance, efficiency and comfort: a review. J Ind Text 2015; 46:1147-1169.

7. Beck B, Blanpain C. Unravelling cancer stem cell potential. Nat Rev Cancer 2013; 13:727-38.

8. Jahangirian H, Lemraski EG, Webster TJ, Rafiee-Moghaddam R, Abdollahi Y. A review of drug delivery systems based on nanotechnology and green chemistry: green nanomedicine. Int J Nanomed 2017; 12:2957-2978.

9. Dilnawaz F, Acharya S, Sahoo SK. Recent trends of nanomedicinal approaches in clinics. Int J Pharm 2018; 538:263-278.

10. Paccez JD, et al. The receptor tyrosine kinase Axl is an essential regulator of prostate cancer proliferation and tumor growth and represents a new therapeutic target. Oncogene 2013; 32:689-98.

11. Rajamani D, Bhasin MK. Identification of key regulators of pancreatic cancer progression through multidimensional systems-level analysis. Genome Med 2016; 8:38.

12. Arredouani MS, et al. Identification of the transcription factor single-minded homologue 2 as a potential biomarker and immunotherapy target in prostate cancer. Clin Cancer Res 2009; 15:5794802.

13. Kumar B, Jalodia K, Kumar P, Gautam HK. Recent advances in nanoparticle-mediated drug delivery. J Drug Deliv Sci Technol 2017; 41:260-268.

14. Jindal $A B$. The effect of particle shape on cellular interaction and drug delivery applications of micro- and nanoparticles. Int J Pharm 2017; 532:450-465.

15. Sundar DS, Antoniraj MG, Kumar CS, Mohapatra SS, Houreld NN Ruckmani K. Recent Trends of Biocompatible and Biodegradable Nanoparticles in Drug Delivery: A Review. Curr Med Chem 2016; 23:3730-3751.

16. Mukherjee B, Dutta L, Mondal L, Dey NS, Chakraborty S, Maji R Shaw TK. Nanoscale Formulations and Diagnostics With Their Recent Trends: A Major Focus of Future Nanotechnology. Curr Pharm Des 2015; 21:5172-86.

17. Tayo LL. Stimuli-responsive nanocarriers for intracellular delivery. Biophys Rev 2017; 9:931-940.

18. Safari J, Zarnegar Z. Advanced drug delivery systems: Nanotechnology of health design A review. J Saudi Chem Soc 2014; 18:8599.

19. Howell M, Wang C, Mahmoud A, Hellermann G, Mohapatra SS Mohapatra S. Dual-function theranostic nanoparticles for drug delivery and medical imaging contrast: perspectives and chal- lenges for use in lung diseases. Drug Deliv Trans Res 2013; 3:352363.

20. Wang C, et al. A chitosan-modified graphene nanogel for noninvasive controlled drug release. Nanomedicine 2013; 9:903-11.

21. Williams EC, Toomey R, Alcantar N. Controlled release niosome embedded chitosan system: effect of crosslink mesh dimensions on drug release. J Biomed Mater Res A 2012; 100:3296-303.

22. Denmark DJ, et al. Remote triggering of thermoresponsive PNIPAM by iron oxide nanoparticles. RSC Advances 2016; 6:56415652.

23. Liu M, Du H, Zhang W, Zhai G. Internal stimuli-responsive nanocarriers for drug delivery: Design strategies and applications. Mater Sci Eng: C 2017; 71:1267-1280.

24. Walsh DP, et al. Bioinspired Star-Shaped Poly(l-lysine) Polypeptides: Efficient Polymeric Nanocarriers for the Delivery of DNA to Mesenchymal Stem Cells. Mol Pharm 2018; 15:1878-1891.

25. Wei Z, et al. The diosgenin prodrug nanoparticles with $\mathrm{pH}$-responsive as a drug delivery system uniquely prevents thrombosis without increased bleeding risk. Nanomedicine 2018; 14:673-684.

26. Boyapalle S, Xu W, Raulji P, Mohapatra S, Mohapatra SS. A Multiple siRNA-Based Anti-HIV/SHIV Microbicide Shows Protection in Both In Vitro and In Vivo Models. PLoS One 2015; 10:e0135288.

27. Lee DW, Shirley SA, Lockey RF, Mohapatra SS. Thiolated chitosan nanoparticles enhance anti-inflammatory effects of intranasally delivered theophylline. Respir Res 2006; 7:112.

28. Lee D, Zhang W, Shirley SA, Kong X, Hellermann GR, Lockey RF, Mohapatra SS. Thiolated chitosan/DNA nanocomplexes exhibit enhanced and sustained gene delivery. Pharm Res 2007; 24:15767.

29. Yang SD, et al. Binary-copolymer system base on low-density lipoprotein-coupled $\mathrm{N}$-succinyl chitosan lipoic acid micelles for co-delivery MDR1 siRNA and paclitaxel, enhances antitumor effects via reducing drug. J Biomed Mater Res B Appl Biomater 2017; 105:1114-1125.

30. Das M, Howell M, Foran EA, lyre R, Mohapatra SS, Mohapatra S Sertoli Cells Loaded with Doxorubicin in Lipid Micelles Reduced Tumor Burden and Dox-Induced Toxicity. Cell Transplant 2017; 26:1694-1702.

31. Zhang Y, Li N, Suh H, Irvine DJ. Nanoparticle anchoring targets immune agonists to tumors enabling anti-cancer immunity without systemic toxicity. Nat Comm 2018; 9:6.

32. Liu L, Ye Q, Lu M, Chen ST, Tseng HW, Lo YC, Ho C. A New Approach to Deliver Anti-cancer Nanodrugs with Reduced Off-target Toxicities and Improved Efficiency by Temporarily Blunting the Reticuloendothelial System with Intralipid. Sci Rep 2017; 7:16106.

33. Germain $M$, et al. Priming the body to receive the therapeutic agent to redefine treatment benefit/risk profile. Sci Rep 2018; 8:4797.

34. Chandan R, Banerjee R. Pro-apoptotic liposomes-nanobubble conjugate synergistic with paclitaxel: a platform for ultrasound responsive image-guided drug delivery. Sci Rep 2018; 8:2624.

35. Hurwitz SN, Nkosi D, Conlon MM, York SB, Liu X, Tremblay DC, Meckes DG, Jr. CD63 Regulates Epstein-Barr Virus LMP1 Exosomal Packaging, Enhancement of Vesicle Production, and Noncanonical NF-kappaB Signaling. J Virol 2017; 91.

36. Hurwitz SN, Rider MA, Bundy JL, Liu X, Singh RK, Meckes DG, Jr. Proteomic profiling of $\mathrm{NCl}-60$ extracellular vesicles uncovers common protein cargo and cancer type-specific biomarkers. Oncotarget 2016; 7:86999-87015.

37. Hurwitz SN, Conlon MM, Rider MA, Brownstein NC, Meckes DG, Jr. Nanoparticle analysis sheds budding insights into genetic drivers of extracellular vesicle biogenesis. J Extracell Vesicles 2016; 5:31295.

38. Minghua $\mathrm{W}$, et al. Plasma exosomes induced by remote ischaemic preconditioning attenuate myocardial ischaemia/reperfusion injury by transferring miR-24. Cell Death Dis 2018; 9:320.

39. Amolegbe SA, et al. Mesoporous silica nanocarriers encapsulated antimalarials with high therapeutic performance. Sci Rep 2018; 8:3078.

40. Mandal T, Beck M, Kirsten N, Linden M, Buske C. Targeting murine leukemic stem cells by antibody functionalized mesoporous silica nanoparticles. Sci Rep 2018; 8:989.

41. Farooq MU, et al. Gold Nanoparticles-enabled Efficient Dual Delivery of Anticancer Therapeutics to HeLa Cells. Sci Rep 2018; 8:2907. 42. Ramalingam V, Varunkumar K, Ravikumar V, Rajaram R. Target de- 
livery of doxorubicin tethered with PVP stabilized gold nanoparticles for effective treatment of lung cancer. Sci Rep 2018; 8:3815.

43. Lian X, Erazo-Oliveras A, Pellois JP, Zhou HC. High efficiency and long-term intracellular activity of an enzymatic nanofactory based on metal-organic frameworks. Nat Comm 2017; 8:2075.

44. Tiwari A, Singh A, Garg N, Randhawa JK. Curcumin encapsulated zeolitic imidazolate frameworks as stimuli responsive drug delivery system and their interaction with biomimetic environment. Sci Rep 2017; 7:12598.

45. Shin CS, Marcano DC, Park K, Acharya G. Application of Hydrogel Template Strategy in Ocular Drug Delivery. Methods Mol Biol 2017; 1570:279-285.

46. Coursey TG, et al. Dexamethasone nanowafer as an effective therapy for dry eye disease. J Control Release 2015; 213:168-174.

47. Chen W, et al. Microneedle-array patches loaded with dual mineralized protein/peptide particles for type 2 diabetes therapy. Nat Comm 2017; 8:1777.

48. Karabin NB, et al. Sustained micellar delivery via inducible transitions in nanostructure morphology. Nat Comm 2018; 9:624.

49. Chaudhari AA, et al. Future Prospects for Scaffolding Methods and Biomaterials in Skin Tissue Engineering: A Review. Int J Mol Sci 2016; 17.

50. Farris AL, Rindone AN, Grayson WL. Oxygen Delivering Biomaterials for Tissue Engineering. J Mater Chem B 2016; 4:3422-3432.

51. Akintewe OO, DuPont SJ, Elineni KK, Cross MC, Toomey RG, Gallant ND. Shape-changing hydrogel surfaces trigger rapid release of patterned tissue modules. Acta Biomater 2015; 11:96-103.

52. Affram $\mathrm{K}, \mathrm{Udofot} \mathrm{O}, \mathrm{Cat} \mathrm{A}$, Agyare $\mathrm{E}$. In vitro and in vivo antitumor activity of gemcitabine loaded thermosensitive liposomal nanoparticles and mild hyperthermia in pancreatic cancer. Int J Adv Res 2015; 3:859-874.

53. Affram K, Udofot O, Agyare E. Cytotoxicity of gemcitabine-loaded thermosensitive liposomes in pancreatic cancer cell lines. Integr Cancer Sci Ther 2015; 2:133-142.

54. Howell M, Mallela J, Wang C, Ravi S, Dixit S, Garapati U, Mohapatra S. Manganese-loaded lipid-micellar theranostics for simultaneous drug and gene delivery to lungs. J Control Release 2013; 167:2108.

55. Martinez JO, et al. Biomimetic nanoparticles with enhanced affinity towards activated endothelium as versatile tools for theranostic drug delivery. Theranostics 2018; 8:1131-1145.

56. Sanchez-Ramos J, et al. Chitosan-Mangafodipir nanoparticles designed for intranasal delivery of siRNA and DNA to brain. J Drug Deliv Sci Technol 2018; 43:453-460.

57. Das M, Wang C, Bedi R, Mohapatra SS, Mohapatra S. Magnetic micelles for DNA delivery to rat brains after mild traumatic brain injury. Nanomedicine 2014; 10:1539-48.

58. Wang C, et al. Dual-purpose magnetic micelles for MRI and gene delivery. J Control Release 2012; 163:82-92.

59. Wang $C$, et al. Multifunctional Chitosan Magnetic-Graphene (CMG) Nanoparticles: a Theranostic Platform for Tumor-targeted Co-delivery of Drugs, Genes and MRI Contrast Agents. J Mater Chem B 2013; 1:4396-4405.

60. Varna M, Xuan HV, Fort E. Gold nanoparticles in cardiovascular imaging. Wiley Interdiscip Rev Nanomed Nanobiotechnol 2018; 10.

61. Liu Y, Zhang P, Li F, Jin X, Li J, Chen W, Li Q. Metal-based NanoEnhancers for Future Radiotherapy: Radiosensitizing and Synergistic Effects on Tumor Cells. Theranostics 2018; 8:1824-1849.

62. Das R, et al. Boosted Hyperthermia Therapy by Combined AC Magnetic and Photothermal Exposures in Ag/Fe3O4 Nanoflowers. ACS Appl Mater Interfaces 2016; 8:25162-9.

63. Usov NA, Nesmeyanov MS, Tarasov VP. Magnetic Vortices as Efficient Nano Heaters in Magnetic Nanoparticle Hyperthermia. Sci Rep 2018; 8:1224.

64. Zhang L, et al. Bioinspired Multifunctional Melanin-Based Nanoliposome for Photoacoustic/Magnetic Resonance Imaging-Guided Efficient Photothermal Ablation of Cancer. Theranostics 2018; 8:1591-1606.

65. Yang G, et al. Smart Nanoreactors for pH-Responsive Tumor Homing, Mitochondria-Targeting, and Enhanced Photodynamic-Immunotherapy of Cancer. Nano letters 2018; 18:2475-2484.

66. Wang $\mathrm{H}$, et al. Targeted production of reactive oxygen species in mitochondria to overcome cancer drug resistance. Nat Comm 2018; 9:562.

67. Mo R, Gu Z. Tumor microenvironment and intracellular signal-ac- tivated nanomaterials for anticancer drug delivery. Materials Today 2016; 19:274-283.

68. Nahire R, et al. Multifunctional polymersomes for cytosolic delivery of gemcitabine and doxorubicin to cancer cells. Biomaterials 2014; 35:6482-6497.

69. Owens EA, et al. Near-Infrared Illumination of Native Tissues for Image-Guided Surgery. J Med Chem 2016; 59:5311-5323.

70. Hiroshima $Y$, et al. Effective fluorescence-guided surgery of liver metastasis using a fluorescent anti-CEA antibody. J Surg Oncol 2016; 114:951-958.

71. Matsumoto T, et al. A Mouse Model of Fluorescent Protein-expressing Disseminated Peritoneal Lymphoma for Fluorescence-guided Surgery. Anticancer Res 2016; 36:4483-7.

72. Wicki A, Witzigmann D, Balasubramanian V, Huwyler J. Nanomedicine in cancer therapy: challenges, opportunities, and clinical applications. J Control Release 2015; 200:138-57.

73. Bernthal NM, et al. Combined in vivo optical and microCT imaging to monitor infection, inflammation, and bone anatomy in an orthopaedic implant infection in mice. J Vis Exp 2014:e51612.

74. Hu Q, Li H, Wang L, Gu H, Fan C. DNA Nanotechnology-Enabled Drug Delivery Systems. Chem Rev 2018.

75. Li J, Green AA, Yan H, Fan C. Engineering nucleic acid structures for programmable molecular circuitry and intracellular biocomputation. Nat Chem 2017; 9:1056-1067.

76. Udomprasert A, Kangsamaksin T. DNA origami applications in cancer therapy. Cancer Sci 2017; 108:1535-1543.

77. Franquelim HG, Khmelinskaia A, Sobczak JP, Dietz H, Schwille P. Membrane sculpting by curved DNA origami scaffolds. Nat Comm 2018; 9:811.

78. Raab M, et al. Using DNA origami nanorulers as traceable distance measurement standards and nanoscopic benchmark structures. Sci Rep 2018; 8:1780.

79. Cronin $M$, et al. High resolution in vivo bioluminescent imaging for the study of bacterial tumour targeting. PLoS One 2012; 7:e30940.

80. Hwang KS, Lee SM, Kim SK, Lee JH, Kim TS. Micro- and nanocantilever devices and systems for biomolecule detection. Annu Rev Anal Chem (Palo Alto Calif) 2009; 2:77-98.

81. Shah P, Zhu X, Zhang X, He J, Li CZ. Microelectromechanical System-Based Sensing Arrays for Comparative in Vitro Nanotoxicity Assessment at Single Cell and Small Cell-Population Using Electrochemical Impedance Spectroscopy. ACS Appl Mater Interfaces 2016; 8:5804-12.

82. Cheemalapati SV, et al. Subcellular and in-vivo Nano-Endoscopy. Sci Rep 2016; 6:34400.

83. Alwarappan S, Cissell K, Dixit S, Mohapatra S, Li CZ. Chitosan-Modified Graphene Electrodes for DNA Mutation Analysis. J Electroanal Chem (Lausanne) 2012; 686:69-72.

84. Girard YK, et al. A 3D fibrous scaffold inducing tumoroids: a platform for anticancer drug development. PLoS One 2013; 8:e75345.

85. Terrell-Hall TB, Ammer AG, Griffith Jl, Lockman PR. Permeability across a novel microfluidic blood-tumor barrier model. Fluids Barriers CNS 2017; 14:3.

86. Samavedi S, Joy N. 3D printing for the development of in vitro cancer models. Curr Opin Biomed Eng 2017; 2:35-42.

87. Vafai N, Lowry TW, Wilson KA, Davidson MW, Lenhert S. Evaporative edge lithography of a liposomal drug microarray for cell migration assays. Nanofabrication 2015; 2:34-42.

88. Kusi-Appiah AE, Lowry TW, Darrow EM, Wilson KA, Chadwick BP, Davidson MW, Lenhert S. Quantitative dose-response curves from subcellular lipid multilayer microarrays. Lab on a chip 2015; 15:3397-404.

89. Ghazanfari L, Lenhert S. Screening of Lipid Composition for Scalable Fabrication of Solvent-Free Lipid Microarrays. Front Mater 2016; 3.

90. Lowry TW, Prommapan P, Rainer Q, Van Winkle D, Lenhert S. Lipid Multilayer Grating Arrays Integrated by Nanointaglio for Vapor Sensing by an Optical Nose. Sensors (Basel, Switzerland) 2015; 15:20863-72.

91. Bazard P, Frisina RD, Walton JP, Bhethanabotla VR. Nanoparticle-based Plasmonic Transduction for Modulation of Electrically Excitable Cells. Sci Rep 2017; 7:7803. 